\title{
The impact of preoperative percutaneous nephrostomy as a treatment strategy before flexible ureteroscopy for impacted upper ureteral stones with hydronephrosis
}

\author{
Go Anan $^{1}$, Daisuke Kudo ${ }^{2}$, Toshimitsu Matsuoka ${ }^{3}$, Yasuhiro Kaiho ${ }^{1}$, Makoto Sato ${ }^{1}$ \\ ${ }^{1}$ Department of Urology, Tohoku Medical and Pharmaceutical University, Sendai, Japan; ${ }^{2}$ Department of Urology, Morioka Municipal Hospital, \\ Morioka, Japan; ${ }^{3}$ Department of Urology, Hachinohe Heiwa Hospital, Hachinohe, Japan \\ Contributions: (I) Conception and design: G Anan, D Kudo, T Matsuoka; (II) Administrative support: Y Kaiho, M Sato; (III) Provision of study \\ materials or patients: G Anan, D Kudo, T Matsuoka; (IV) Collection and assembly of data: G Anan, D Kudo, T Matsuoka; (V) Data analysis and \\ interpretation: G Anan; (VI) Manuscript writing: All authors; (VII) Final approval of manuscript: All authors. \\ Correspondence to: Go Anan, MD, PhD. Department of Urology, School of Medicine, Tohoku Medical and Pharmaceutical University, Sendai, Miyagi \\ 983-8536, Japan. Email: goanan@tohoku-mpu.ac.jp.
}

Background: The treatment of impacted upper ureteral stones with hydronephrosis remains a challenge for urologists. The current study aimed to evaluate the impact of preoperative percutaneous nephrostomy (PNS) as a treatment strategy before flexible ureteroscopy (f-URS) of asymptomatic impacted upper ureteral stones with hydronephrosis.

Methods: This multicenter retrospective study included patients who underwent PNS (group A, n=61) and those who did not (group B, n=75) before f-URS for asymptomatic impacted upper ureteral stones with hydronephrosis. Impacted ureteral stones are defined as those that remain in one position for $>2$ months. Operative outcomes, including stone-free rate, operation time, postoperative hospital days, and complication rate, were evaluated.

Results: There were no significant differences in age, sex, and stone size between the two groups except in the grade of hydronephrosis, with group A having more cases of advanced hydronephrosis than group B. The stone-free rate was significantly higher in group A than in group B [95\% vs. 77\% $(\mathrm{P}=0.004)]$. However, there were no significant differences between the groups in operation time [55 vs. $55 \mathrm{~min}(\mathrm{P}=0.84)$, postoperative fever [5\% vs. $5 \%(\mathrm{P}=1.00)$ ], and postoperative hospital days [2 vs. 2 days $(\mathrm{P}=0.44)]$. In group A, preoperative PNS placement was performed 4 days before $\mathrm{f}-\mathrm{URS}$, and the PNS was removed postoperatively on the same day of the f-URS. Additionally, subgroup analysis was performed in cases of grade 2 and 3 hydronephrosis. A total of 110 patients, 60 who underwent f-URS with PNS and 50 who underwent $\mathrm{f}$-URS without PNS, were included. The stone-free rate was significantly higher in f-URS with PNS than in f-URS without PNS [95\% vs. $76 \%(\mathrm{P}=0.005)$ ]. However, no significant differences were found between the groups in operation time, ureteral injury, postoperative fever, and postoperative hospital days.

Conclusions: At grade 2 or 3 hydronephrosis, preoperative PNS as a treatment strategy for a few days prior to f-URS for impacted upper ureteral stones improved the stone-free rate without increasing the operation time and postoperative length of hospital stay.

Keywords: Impacted stone; hydronephrosis; lithotripsy; percutaneous nephrostomy (PNS); ureteroscopy

Submitted Jun 21, 2021. Accepted for publication Sep 02, 2021.

doi: $10.21037 /$ tau-21-547

View this article at: https://dx.doi.org/10.21037/tau-21-547

\footnotetext{
^ ORCID: 0000-0001-8325-2606.
} 


\section{Introduction}

The lifetime risk of urolithiasis is $8-19 \%$ in males and $3-5 \%$ in females $(1,2)$. Furthermore, the recurrence rate of urolithiasis has been reported to be as high as $50 \%$ at 5 years and $80-90 \%$ at 10 years $(3,4)$. Due to technological advances, such as ureteral access sheath, holmium laser, and stone basket, which enable the management of intrarenal stones, flexible ureteroscopy (f-URS) has been established as a minimally invasive modality to treat intrarenal calculi (5-7). Impacted ureteral stones are defined as those that remain in one position for $>2$ months (8). Long-term impacted ureteral stones may result in pain and urinary tract infections and further may lead to asymptomatic hydronephrosis in some cases. One of the most difficult-toremove stones in f-URS is the impacted ureteral stone with hydronephrosis.

In patients with impacted ureteral stones, preoperative ureteral stent itself might be difficult owing to ureter impaction, ureteral polyps, and ureter strictures, and in some cases, there might be a risk of ureteral injury. The advantages of preoperative percutaneous nephrostomy (PNS) are straightening of the ureter owing to the elevation of the kidney as a result of the decompression of hydronephrosis and reduced intrarenal pressure by drainage without risk of ureteral injury, particularly when the ureteral stone is the impacted stone. Kwon et al. reported that f-URS with PNS utilized due to urinary tract infection or pain might improve stone-free rate without increasing operation time or complication rate compared to f-URS without PNS (9). However, in this report, PNS was performed owing to symptomatic ureteral stone causing urinary tract infection or pain for approximately 1 month prior to f-URS. To the best of our knowledge, this is the first report on preoperative PNS as a treatment strategy before f-URS for impacted upper ureteral stones and its impact on stone-free rate and perioperative outcomes. Here, we compared the operative result and complication rate between the patients undergoing preoperative PNS and those not undergoing PNS for asymptomatic impacted upper ureteral stones. We present the following article in accordance with the STROBE reporting checklist (available at https://dx.doi. org/10.21037/tau-21-547).

\section{Methods}

The study was conducted in accordance with the Declaration of Helsinki (as revised in 2013). The study was approved by the Institutional Ethics Committees of Tohoku Medical and Pharmaceutical University Hospital School of Medicine (Protocol 2-056), Morioka Municipal Hospital (Protocol 069), and Hachinohe Heiwa Hospital (Protocol 21-02). Individual consent for this retrospective analysis was waived.

\section{Study population}

This retrospective study investigated treatment outcomes in patients with asymptomatic impacted upper ureteral stones and hydronephrosis who underwent f-URS between May 2016 and March 2020 in Tohoku Medical and Pharmaceutical University Hospital, Morioka Municipal Hospital, and Hachinohe Heiwa Hospital in Japan. All consecutive patients were included in the analysis without any exclusion to prevent potential selection bias. The patients were divided into those who underwent PNS (group $\mathrm{A}, \mathrm{n}=61$ ) and those who did not undergo PNS (group B, $\mathrm{n}=75$ ) before $\mathrm{f}$-URS. The inclusion criteria included (I) age $\geq 18$ years and (II) impacted ureteral stone defined as one that stayed in the same location for at least 2 months and resulted in ureter obstruction (8). The exclusion criteria included (I) ureteral stones sized $\geq 2.0 \mathrm{~cm}$; (II) lower ureteral stones; (III) stones associated with symptoms such as pyelonephritis, pain, renal dysfunction, and urinary fistula that needed immediate drainage; (IV) impacted ureteral stone without hydronephrosis; (V) preoperative implantation of ureteral stent for the resolution of urinary tract obstruction; and (VI) malformations in the urinary tract, such as cases after urinary diversion. The criteria to decide which type of procedure (with PNS or without PNS) to perform was the patient's and surgeon's decision.

The primary outcome measure was stone-free status, defined as a stone size of $\leq 2 \mathrm{~mm}$ measured on computed tomography (CT) at 1 month after f-URS. The secondary outcome measure was intraoperative and postoperative complications such as intraoperative ureteral injury, postoperative hydronephrosis, and postoperative fever. To investigate the efficacy, we compared the results between f-URS with PNS (group A) and f-URS without PNS (group B).

In group A, PNS was employed as the treatment of f-URS for few days before the operation. Because a large number of referrals for patients with urolithiasis are made to our institutions, certain number of patients have a long history of stones since their initial diagnosis. Comparing the images from the referring hospitals with those from 

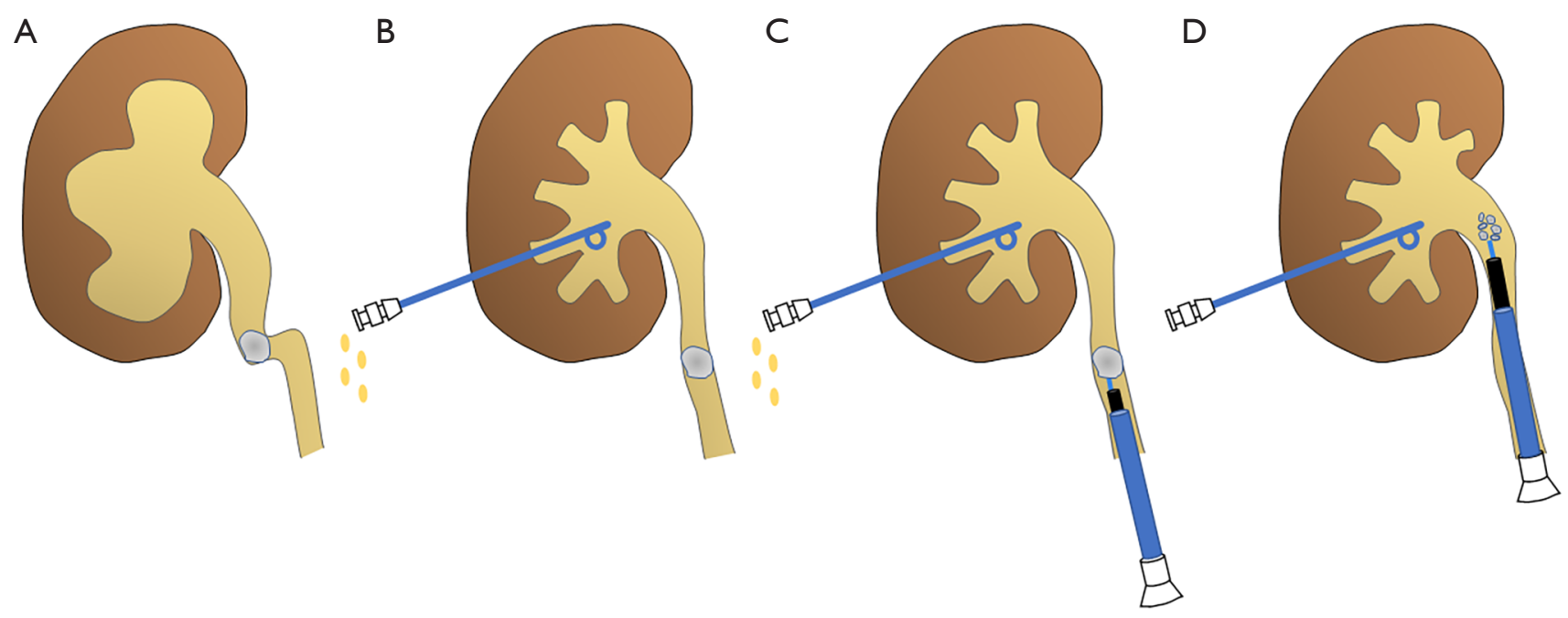

Figure 1 Diagram of the management strategy. (A) Impacted upper ureteral stone with hydronephrosis. (B) Preoperative PNS improves hydronephrosis and ureteral flexion. (C) PNS reduces intrarenal pressure during lithotripsy. (D) PNS closure maintains the intrarenal space during stone retrieval by f-URS. PNS, percutaneous nephrostomy; f-URS, flexible ureteroscopy.

our institutions, we continuously assessed the presence of impacted ureteral stones for more than 2 months. In our institutions, preoperative ureteral stent before f-URS is not performed for long-term impacted stones with hydronephrosis because of the possibility of ureteral injury; therefore, the two groups were compared.

The preoperative clinical information included age, sex, body mass index $\left(\mathrm{kg} / \mathrm{m}^{2}\right)$, stone size $(\mathrm{mm})$, stone laterality (right or left), and CT attenuation value in Hounsfield units. Perioperative clinical information included total operation time ( $\mathrm{min})$, total laser energy $(\mathrm{KJ})$, and complications such as ureteral injury and macrohematuria. The perioperative ureteral injury was determined based on intraoperative ureteroscopic findings and evaluated using the post-ureteroscopic lesion scale (10). Postoperative clinical information included stone-free status, defined as a stone size of $\leq 2 \mathrm{~mm}$ by CT at 1 month after f-URS, postoperative fever, and postoperative hospitalization days. Postoperative fever was defined as a body temperature above $38.0^{\circ} \mathrm{C}$. The hydronephrosis grade was evaluated by preoperative CT and classified into three grades: grade 1 means mild dilatation such as pelvic dilatation only; grade 2 means moderate dilatation of the urinary system; and grade 3 means severe dilatation of the urinary system (11). Grade 1 hydronephrosis was defined as mild hydronephrosis, grade 2 hydronephrosis as moderate hydronephrosis, and grade 3 hydronephrosis as severe hydronephrosis. The grade of postoperative hydronephrosis was determined using CT. The definition of postoperative ureteral stenosis was the need for ureteral stenosis release or ureteral stent reimplantation owing to residual severe hydronephrosis.

\section{Surgical technique}

The surgeries were mainly performed by three experienced surgeons at our institutions. All patients underwent a standard surgical procedure (Figures 1,2) (12). In group A, a PNS (8 or $10 \mathrm{Fr}$ ) was inserted by urologists, under local anesthesia and fluoroscopy, after the patients' admission to the hospital and retained for a median of 4 days (range, 2-6 days) before $\mathrm{f}$-URS (Figure 1B). Because the basic purpose is drainage and preservation of the possibility of antegrade approach, a nephrostomy was mostly placed in the middle or lower calyx. All f-URS procedures were performed under general anesthesia using a semirigid ureteroscope (6.0/7.5 or 8.0/9.8 Fr; Wolf, Knittlingen, Germany or 6.4/7.8 Fr; Olympus, Tokyo, Japan), a flexible ureteroscope (7.5 Fr Flex-X2; Karl Storz, Tuttlingen, Germany or 7.95 Fr URF-P6; Olympus), and a holmium laser lithotripsy system (100 or $120 \mathrm{~W}$ holmium: yttrium aluminum garnet laser; VersaPulse PowerSuite; Lumenis, Yokneam, Israel). A ureteral access sheath was placed in all patients (size, $11 / 13$ or $12 / 14 \mathrm{Fr}$ ). A 1.5 or $1.7 \mathrm{Fr}$ tipless nitinol basket was routinely used for stone removal (Figure 2E). Briefly, the PNS was opened and the intrarenal pressure was reduced from the initiation of f-URS until the impacted ureteral stone was crushed and pushed back into the renal space (Figure 1C). 

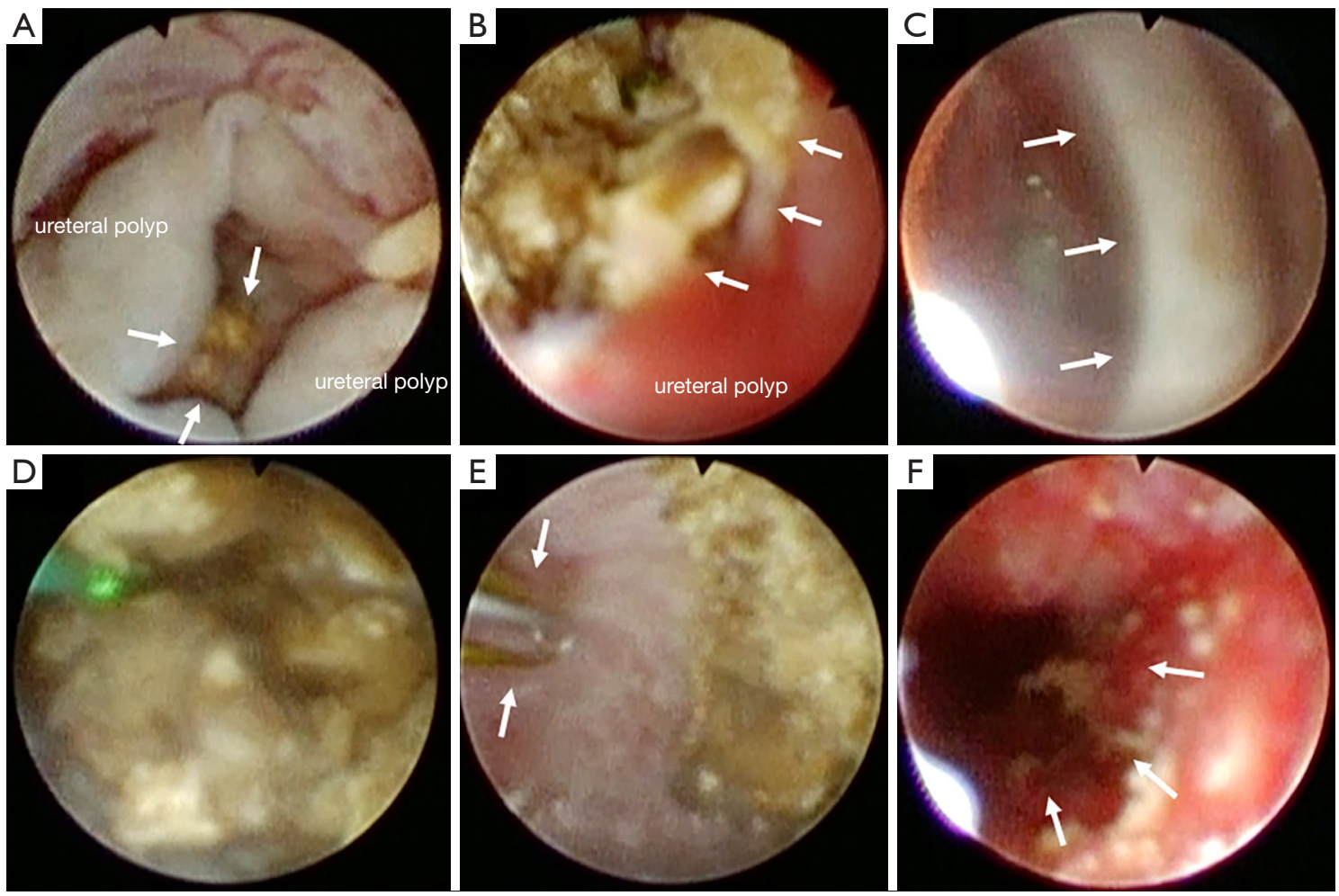

Figure 2 Representative intraoperative images of preoperative PNS and f-URS. (A) View of the impacted ureteral stone by f-URS. The white arrows indicate the impacted stone surrounded by ureteral polyp. (B) View of fragmentation of the impacted stone in the ureter. The white arrows indicate the impacted stone. (C) View of the PNS from the intrarenal space by flexible ureteroscope. The white arrows indicate the PNS. (D) View of the stone fragmentation in the renal pelvis. (E) View of the stone removal by a basket catheter. The white arrows indicate the basket catheter. (F) View of the removal of the impacted ureteral stone at the end of f-URS. The white arrows indicate the ureter view after removal of the impacted stone. PNS, percutaneous nephrostomy; f-URS, flexible ureteroscopy.

Next, the PNS was clamped at the time of retrieval of stone fragments in the renal space (Figure 1D). Finally, the PNS was removed, and a ureteral stent was inserted in all the cases to prevent ureteral stricture caused by the impacted ureteral stones. The ureteral stent was removed 2 weeks after $\mathrm{f}$-URS.

\section{Statistical analysis}

Patient characteristics were described using medians with ranges for continuous variables. Statistical analyses were performed using the JMP statistical software version 12.2 (SAS Institute, Cary, NC. USA). Intergroup differences in continuous variables were assessed using the Mann-Whitney $\mathrm{U}$ test, and categorical variables were assessed using the chi-squared test and Fisher's exact test. Three groups were assessed using the Kruskal-Wallis test. Multivariate analysis was conducted using the logistic regression analysis. $\mathrm{P}$ value of $<0.05$ was considered statistically significant.

\section{Results}

A total of 136 patients, comprising of 61 patients who underwent f-URS with PNS (group A) and 75 patients who underwent f-URS without PNS (group B), were included in the study. There was a significant difference between the two groups with respect to the grade of hydronephrosis $(\mathrm{P}=0.001)$. Of the patients in groups A and B, 1 and 25 had grade 1 hydronephrosis, 46 and 37 had grade 2 hydronephrosis, and 14 and 13 had grade 3 hydronephrosis, respectively. However, there were no differences in background characteristics, including age, sex, body mass index, stone size, stone laterality, and CT attenuation value, between the two groups (Table 1). In group A, the median duration of preoperative nephrostomy tube placement was 4 days (range, 2-6 days) and all patients had the nephrostomy tube removed on the same day of f-URS after the conclusion of the procedure. The location of the nephrostomy was the middle calyx in 
Table 1 Patient and stone characteristics between the groups

\begin{tabular}{lccc}
\hline Group & URS with PNS $(\mathrm{n}=61)$ & URS without PNS $(\mathrm{n}=75)$ & P value \\
\hline Age (years) & $63[34-88]$ & $61[35-86]$ & 0.17 \\
Sex (male) & $48[79]$ & $51[68]$ & 0.16 \\
Body mass index $\left(\mathrm{kg} / \mathrm{m}^{2}\right)$ & $24[16-31]$ & $25[15-35]$ & 0.23 \\
Stone size $(\mathrm{mm})$ & $12[7-19]$ & $12[6-19]$ & $39[52]$ \\
Stone laterality (right) & $38[62]$ & $1,260[450-1,840]$ & 0.18 \\
CT value (HU) & $1,350[350-1,960]$ & & 0.11 \\
Hydronephrosis & & $25[33]$ & 0.001 \\
Grade 1 & $1[2]$ & $37[49]$ & $13[18]$ \\
Grade 2 & $46[75]$ & $14[23]$ & \\
Grade 3 & & & \\
\hline
\end{tabular}

Date are presented as median [range] or n [\%]. CT, Computed tomography; URS, ureteroscopy; PNS, percutaneous nephrostomy.

Table 2 Comparison of operative outcome and complications between the groups

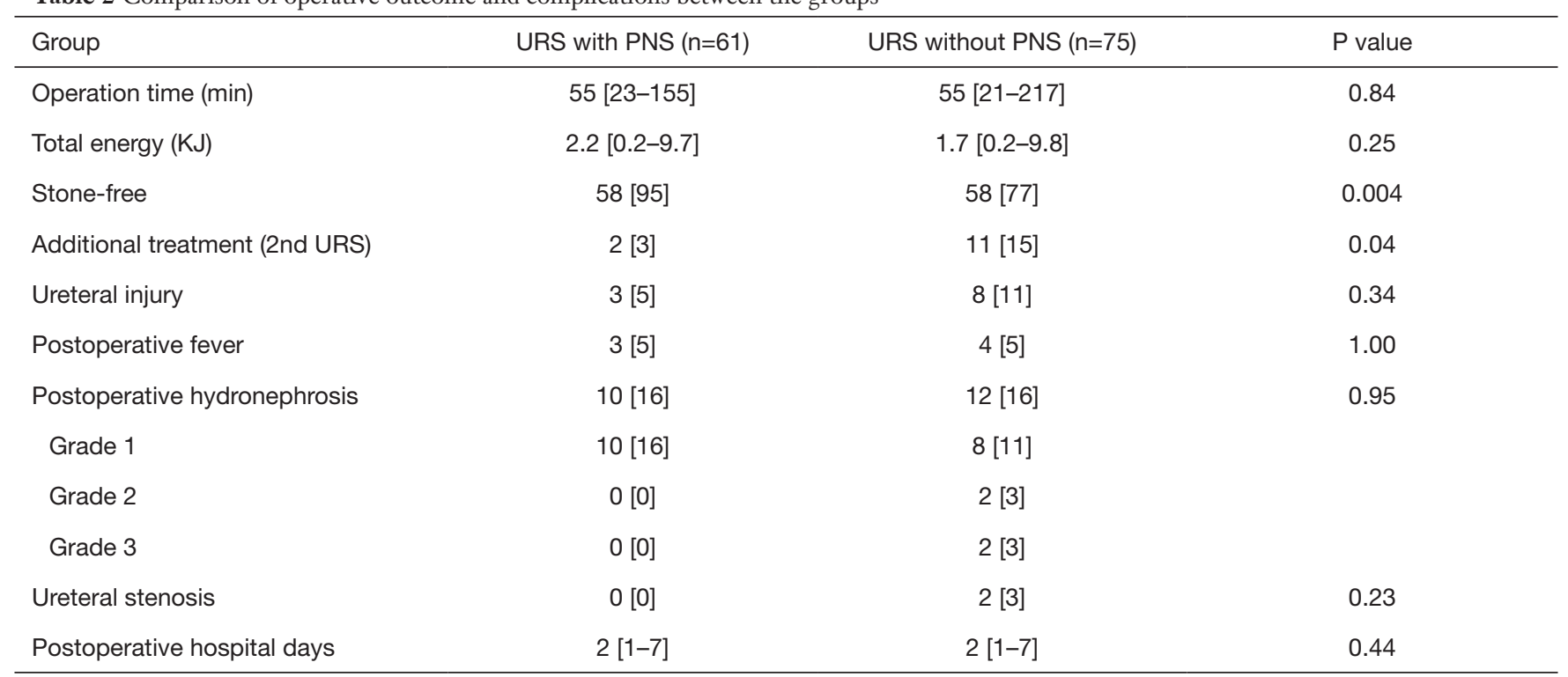

Date are presented as median [range] or $\mathrm{n}[\%]$. URS, ureteroscopy; PNS, percutaneous nephrostomy.

32 patients and the lower calyx in 29 patients.

Table 2 shows the operative outcomes. The stone-free rate was significantly higher in group A than in group B (95\% vs. $77 \% ; \mathrm{P}=0.004$ ). Moreover, the rate of 2 nd URS as additional treatment was significantly lower in group A than in group B ( $3 \%$ vs. $15 \% ; \mathrm{P}=0.04)$. However, there were no significant differences between groups $\mathrm{A}$ and $\mathrm{B}$ regarding operation time ( 55 vs. $55 \mathrm{~min} ; \mathrm{P}=0.84)$ and total laser energy ( 2.2 vs. $1.7 \mathrm{~kJ} ; \mathrm{P}=0.25)$. Furthermore, there were no significant differences between groups $\mathrm{A}$ and $\mathrm{B}$ in ureteral injury ( $5 \%$ vs. $11 \% ; \mathrm{P}=0.34$ ), postoperative fever ( $5 \%$ vs. $5 \% ; \mathrm{P}=1.00)$, postoperative hydronephrosis $(16 \%$ vs. $16 \% ; \mathrm{P}=0.95)$, postoperative ureteral stenosis $(0 \%$ vs. $3 \% ; \mathrm{P}=0.23$ ), and postoperative hospital days ( 2 s. 2 days; $\mathrm{P}=0.44)$. Among the complications, the post-ureteroscopic lesion scale of ureteral injury grade 1,2, and 3 occurred in 1,1 , and 1 patient, respectively, in group A and 6,0 , and 2 patients, respectively, in group B. The ureteral injuries 
Table 3 Univariate and multivariate analyses of associations between various parameters and stone-free

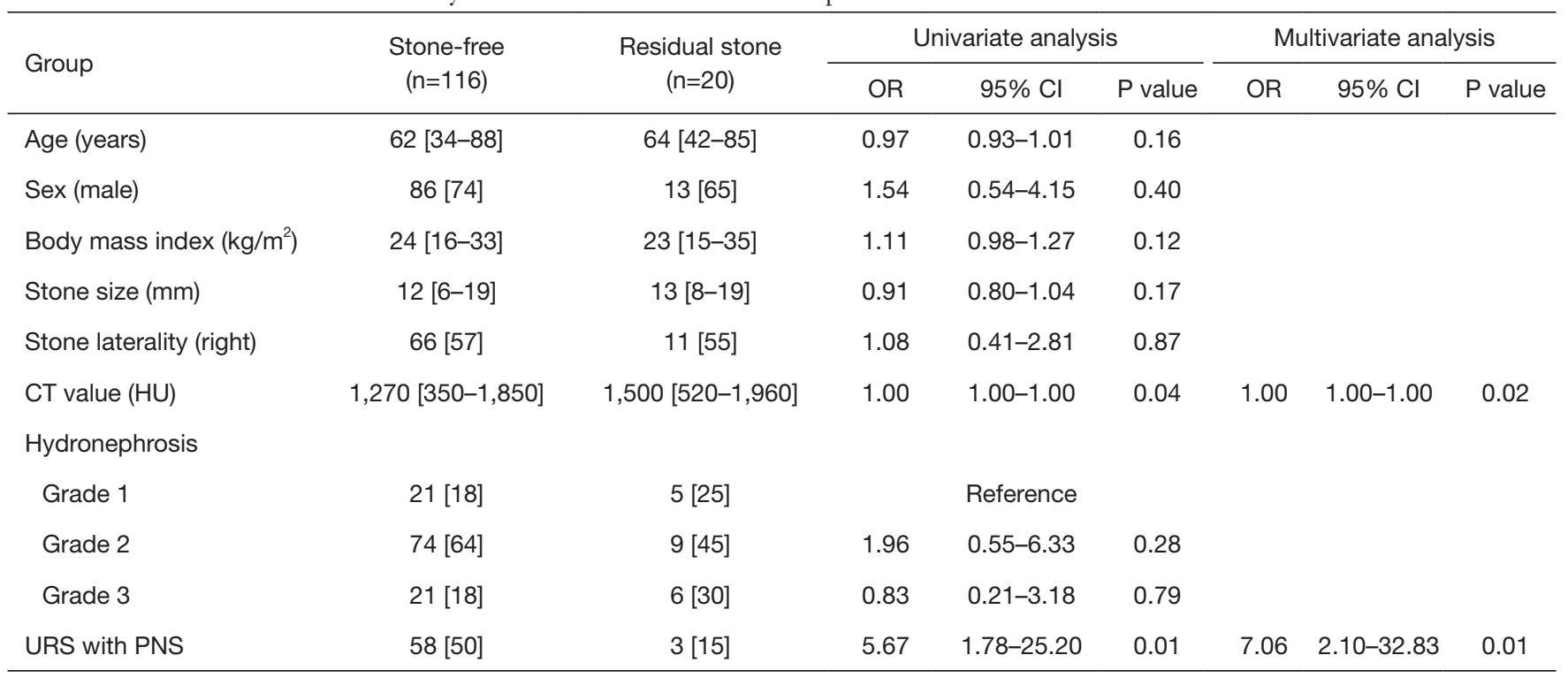

Date are presented as median [range] or $\mathrm{n}$ [\%]. CT, computed tomography; URS, ureteroscopy; PNS, percutaneous nephrostomy; OR, odds ratio; $\mathrm{Cl}$, confidence interval.

occurred mostly by not intraoperative ureteroscopy or laser manipulation but the damage to the ureteral mucosa after the removal of the impacted ureteral stones; in a few cases, the ureteral injuries occurred because of the access sheath or missed shots by the laser. No patient experienced gross hematuria necessitating hemostatic surgery or blood transfusion due to postoperative blood loss.

Table 3 shows the preoperative predictive factors for stone-free and residual stone. In the univariate analysis, URS with PNS [odds ratio (OR), 5.67; $\mathrm{P}=0.01$ ] and CT value $(\mathrm{OR}, 1.00 ; \mathrm{P}=0.04)$ were significant preoperative factors of stone-free. In the multivariate analysis, URS with PNS (OR, 7.06; $\mathrm{P}=0.01)$ and $\mathrm{CT}$ value $(\mathrm{OR}, 1.00 ; \mathrm{P}=0.02)$ were significant preoperative factors of stone-free.

Since grade 1 hydronephrosis cases were few in Group A, subgroup analysis was performed only for grade 2 and 3 hydronephrosis cases. A total of 110 patients, 60 who underwent $\mathrm{f}$-URS with PNS and 50 who underwent f-URS without PNS, were included in the subgroup analysis. Table 4 shows the patient background and operative outcomes. No differences were observed in background characteristics, including age, sex, body mass index, stone size, and CT attenuation value, between the two groups. The stone-free rate was significantly higher in $\mathrm{f}$-URS with PNS than in f-URS without PNS (95\% vs. 76\%; $\mathrm{P}=0.005$ ). However, no significant differences were found between the groups in operation time ( 55 vs. $65 \mathrm{~min} ; \mathrm{P}=0.29)$, ureteral injury ( $5 \%$ vs. $14 \% ; \mathrm{P}=0.18)$, postoperative fever $(3 \%$ vs. $8 \% ; \mathrm{P}=0.41)$, postoperative hydronephrosis $(17 \%$ vs. $16 \% ; \mathrm{P}=0.93)$, and postoperative hospital days ( 2 s. 2 days; $\mathrm{P}=0.10)$.

\section{Discussion}

In patients with urolithiasis, treatment of impacted ureteral stones with hydronephrosis is particularly challenging. Regarding the f-URS-based treatment strategy for impacted ureteral stones, there are some reports on preoperative ureteral stent; however, no reports are available on preoperative nephrostomy as a treatment strategy for URS. Therefore, in this study that focuses on the treatment of impacted upper ureteral stones and hydronephrosis, we found that using preoperative PNS as a treatment strategy for a few days before f-URS might be associated with better stone-free rate in the absence of increases in operation time and postoperative hospital days.

Generally, f-URS is a minimally invasive procedure, which has a good acceptance by patients, and most patients recover quickly after the operation. However, it was reported that the stone-free rate was $82 \%$ by f-URS in patients with impacted ureteral stones compared with the stone-free rate of $94.6 \%$ in patients without impacted ureteral stones (13). Impaction was also reported 
Table 4 Comparison of patient background and operative outcome between URS with PNS and URS without PNS groups in patients with grade 2 and 3 of hydronephrosis

\begin{tabular}{|c|c|c|c|}
\hline Group & URS with PNS $(n=60)$ & URS without PNS $(n=50)$ & $P$ value \\
\hline Age (years) & 63 [34-88] & 60 [35-86] & 0.10 \\
\hline Sex (male) & $47[78]$ & $32[64]$ & 0.10 \\
\hline Body mass index $\left(\mathrm{kg} / \mathrm{m}^{2}\right)$ & $24[16-31]$ & 25 [18-35] & 0.09 \\
\hline Stone size $(\mathrm{mm})$ & $12[7-19]$ & 12 [6-19] & 0.63 \\
\hline Stone laterality (right) & 37 [62] & $27[54]$ & 0.42 \\
\hline CT value $(\mathrm{HU})$ & $1,350[350-1,960]$ & $1,220[500-1,840]$ & 0.07 \\
\hline Hydronephrosis & & & 0.75 \\
\hline Grade 2 & $46[77]$ & $37[74]$ & \\
\hline Grade 3 & 14 [23] & $13[26]$ & \\
\hline Operation time (min) & 55 [23-155] & $65[24-217]$ & 0.29 \\
\hline Total energy (KJ) & $2.2[0.2-9.7]$ & $1.8[0.2-9.8]$ & 0.50 \\
\hline Stone-free & 57 [95] & $38[76]$ & 0.005 \\
\hline Additional treatment (2nd URS) & $2[3]$ & $8[16]$ & 0.04 \\
\hline Ureteral injury & $3[5]$ & $7[14]$ & 0.18 \\
\hline Postoperative fever & $2[3]$ & $4[8]$ & 0.41 \\
\hline Postoperative hydronephrosis & $10[17]$ & $8[16]$ & 0.93 \\
\hline Grade 1 & $10[17]$ & $5[10]$ & \\
\hline Grade 2 & 0 & 1 [2] & \\
\hline Grade 3 & 0 & $2[4]$ & \\
\hline Ureteral stenosis & $0[0]$ & 1 [2] & 0.24 \\
\hline Postoperative hospital days & $2[1-7]$ & $2[1-7]$ & 0.10 \\
\hline
\end{tabular}

Date are presented as median [range] or $\mathrm{n}$ [\%]. CT, Computed tomography; URS, ureteroscopy; PNS, percutaneous nephrostomy.

to significantly correlate with the stone-free rate (14). Treatment approaches for impacted ureteral stones include f-URS, percutaneous nephrolithotomy, shock wave lithotripsy, and laparoscopic ureterolithotomy $(15,16)$. Compared with other treatments, f-URS is a less invasive treatment for impacted ureteral stones, although there are several disadvantages with $f-U R S$ for impacted ureteral stones. First, the stone-free rate is relatively low. During $\mathrm{f}-\mathrm{URS}$, the stone and its debris are inclined to return to the renal pelvis by the flushing fluid, resulting in residual stones (14). Second, complications such as ureteral injury and postoperative ureteral stenosis are relatively high with f-URS performed for impacted ureteral stones $(15,17)$. Several studies reported that intraoperative complication rates were higher with $\mathrm{f}$-URS in patients with impacted stones compared to those without impacted stones $(7.9 \%$ vs. $3.0 \% ; \mathrm{P}<0.001)(13,14)$.

The present study aimed to determine whether preoperative PNS could increase stone-free rate for impacted ureteral stones with hydronephrosis. Usually, ureteral polyps occur immediately distal to the impacted ureteral stone (18), and ureteral polyps and strictures make it difficult to achieve a secure view of the ureteral lumen (Figure 2A). Additionally, ureteral flexion due to long-term hydronephrosis hinders endoscopy in several aspects, including manipulation of the flexible ureteroscope, securing a visual field of the ureteral lumen, and straightening of the flexed ureter (Figure 1A). In the present study, the stone-free rate was significantly higher in the preoperative PNS group compared to the group 
without preoperative PNS. One advantage of preoperative PNS might be straightening of the flexed ureter caused by the elevation of the kidney following the decompression of hydronephrosis. In the case of impacted ureteral stone, the pressure above the stone could not be relieved sufficiently; however, presence of the PNS catheter facilitates efficient irrigation as well as the achievement of a clear and safe view of the surgical field, which may result in a better stonefree rate. Until the impacted ureteral stone was pushed back into the renal pelvis or it was penetrated, preoperative nephrostomy might be useful to reduce the intrarenal pressure by drainage. Additionally, adequate irrigation could prevent ureteral damage due to high temperatures associated with laser use during f-URS (19).

In the present study, we also determined whether preoperative PNS for impacted ureteral stones with hydronephrosis was associated with complications such as ureteral injury and postoperative fever. Intrarenal pressure tends to increase in the presence of impacted ureteral stones with hydronephrosis; therefore, postoperative fever and the presence of hypertrophy and secretion of an adhesive fibrinous exudate have been described in patients with stone impaction $(18,20)$. Pathologic changes such as ureteral polyps immediately distal to the impacted ureteral stone and ureteral edematous changes can lead to further challenges in retrograde access to the stone, greater adherence of stone fragments to the mucosa, and increased risk of bleeding, perforation, stricture formation, and repeat operation $(21,22)$. A clinical report of 2,650 patients undergoing ureteroscopic treatment of impacted stones demonstrated higher rates of bleeding, ureteral avulsion, and ureteral perforation (14). As a result, surgeons may encounter challenges in accessing the stone, more bleeding from the mucosa, and difficulty in freeing the stone from the area of impaction as well as a greater chance of ureteral perforation (8). In the study, there were no significant differences with or without preoperative PNS in ureteral injury, postoperative fever, postoperative hydronephrosis, postoperative ureteral stenosis, and postoperative hospital days.

Alternatively, there are some reports on preoperative ureteral stent for ureteral stones (23-27). Preoperative ureteral stent under fluoroscopy for impacted ureteral stones can potentially lead to stent failure and complications, such as ureteral injury and urinary tract infection. Furthermore, preoperative ureteral stents are associated with pain, symptoms of urinary irritation, and hematuria, which can significantly impact the patient's quality of life (23). However, preoperative ureteral stent before URS has been reported to increase stone-free rate due to ureteral dilation $(24,25)$. In contrast, several studies have reported that preoperative ureteral stent before URS does not impact the stone-free rate and operation time $(26,27)$. Therefore, the impact of preoperative ureteral stent for impacted stone remains controversial. Additionally, the preoperative ureteral stent has to be removed at the start of $\mathrm{f}$-URS and therefore could not provide direct drainage during intraoperative lithotripsy, whereas the PNS could provide direct drainage during intraoperative lithotripsy; therefore, PNS could be effective for intraoperative control of intrarenal pressure.

There are several limitations in the present study. First, this is a retrospective study and future prospective studies under strictly defined conditions are necessary to confirm the utility of preoperative PNS before f-URS for asymptomatic impacted upper ureteral stones. In this study, there was a significant difference between the two groups with respect to the grade of hydronephrosis. Second, the patients were treated at three different hospitals by different surgeons, and the selection of preoperative PNS was at the surgeon's discretion. Third, the present study did not compare the impact of PNS performed at the same time of $\mathrm{f}-\mathrm{URS}$ with that of PNS performed prior to f-URS and did not evaluate the timing of preoperative PNS. Fourth, the present study did not compare preoperative PNS with preoperative ureteral stent; therefore, it is impossible to determine which treatment is more effective. Fifth, in this study, differences in the quality of life between patients with and without PNS was not assessed. However, the current study findings suggest that preoperative PNS as a treatment strategy for a few days prior to f-URS for asymptomatic impacted upper ureteral stones might be an effective surgical approach for f-URS because of high stone-free rate, particularly when the grade of hydronephrosis was moderate or severe.

\section{Conclusions}

At grade 2 or 3 hydronephrosis, preoperative PNS as a treatment strategy for a few days before f-URS for impacted upper ureteral stones improves the stone-free rate without increasing the operation time, ureteral injury, and postoperative length of hospital stay. Preoperative PNS as a treatment strategy for a few days prior to f-URS might be an effective preoperative treatment for f-URS of impacted 
ureteral stones.

\section{Acknowledgments}

We would like to thank for the support and assistance provided by the entire staff of the Department of Urology, Tohoku Medical and Pharmaceutical University School of Medicine, Morioka Municipal Hospital, and Hachinohe Heiwa Hospital. We would also like to thank Yuri Anan for creating the graphic illustration.

Funding: None.

\section{Footnote}

Reporting Checklist: The authors have completed the STROBE reporting checklist. Available at https://dx.doi. org/10.21037/tau-21-547

Data Sharing Statement: Available at https://dx.doi. org/10.21037/tau-21-547

Conflicts of Interest: All authors have completed the ICMJE uniform disclosure form (available at https://dx.doi. org/10.21037/tau-21-547). The authors have no conflicts of interest to declare.

Ethical Statement: The authors are accountable for all aspects of the work in ensuring that questions related to the accuracy or integrity of any part of the work are appropriately investigated and resolved. The study was conducted in accordance with the Declaration of Helsinki (as revised in 2013). The study was approved by the Institutional Ethics Committees of Tohoku Medical and Pharmaceutical University Hospital School of Medicine (2-056), Morioka Municipal Hospital (Protocol 069), and Hachinohe Heiwa Hospital (Protocol 21-02), and individual consent for this retrospective analysis was waived.

Open Access Statement: This is an Open Access article distributed in accordance with the Creative Commons Attribution-NonCommercial-NoDerivs 4.0 International License (CC BY-NC-ND 4.0), which permits the noncommercial replication and distribution of the article with the strict proviso that no changes or edits are made and the original work is properly cited (including links to both the formal publication through the relevant DOI and the license). See: https://creativecommons.org/licenses/by-nc-nd/4.0/.

\section{References}

1. Trinchieri A. Epidemiology of urolithiasis: an update. Clin Cases Miner Bone Metab 2008;5:101-6.

2. Romero V, Akpinar H, Assimos DG. Kidney stones: a global picture of prevalence, incidence, and associated risk factors. Rev Urol 2010;12:e86-96.

3. Uribarri J, Oh MS, Carroll HJ. The first kidney stone. Ann Intern Med 1989;111:1006-9.

4. Khan A. Prevalence, pathophysiological mechanisms and factors affecting urolithiasis. Int Urol Nephrol 2018;50:799-806.

5. Wetherell DR, Ling D, Ow D, et al. Advances in ureteroscopy. Transl Androl Urol 2014;3:321-7.

6. Somani BK, Ploumidis A, Pappas A, et al. Pictorial review of tips and tricks for ureteroscopy and stone treatment: an essential guide for urologists from PETRA research consortium. Transl Androl Urol 2019;8:S371-80.

7. Anan G, Komatsu K, Hatakeyama S, et al. One-surgeon basketing technique for stone extraction during flexible ureteroscopy for urolithiasis: A comparison between novice and expert surgeons. Int J Urol 2020;27:1072-7.

8. Roberts WW, Cadeddu JA, Micali S, et al. Ureteral stricture formation after removal of impacted calculi. J Urol 1998;159:723-6.

9. Kwon SY, Choi JY, Kim BS, et al. Efficacy of percutaneous nephrostomy tube during ureteroscopy for upper ureter stone management. J Endourol 2013;27:1209-12.

10. Traxer O, Thomas A. Prospective evaluation and classification of ureteral wall injuries resulting from insertion of a ureteral access sheath during retrograde intrarenal surgery. J Urol 2013;189:580-4.

11. Cho KS, Hong SJ, Cho NH, et al. Grade of hydronephrosis and tumor diameter as preoperative prognostic factors in ureteral transitional cell carcinoma. Urology 2007;70:662-6.

12. Anan G, Hattori K, Hatakeyama S, et al. Efficacy of onesurgeon basketing technique for stone extraction during flexible ureteroscopy for urolithiasis. Arab J Urol 2021. DOI: 10.1080/2090598X.2021.1889943.

13. Seitz C, Tanovic E, Kikic Z, et al. Impact of stone size, location, composition, impaction, and hydronephrosis on the efficacy of holmium:YAG-laser ureterolithotripsy. Eur Urol 2007;52:1751-7.

14. Legemate JD, Wijnstok NJ, Matsuda T, et al. Characteristics and outcomes of ureteroscopic treatment in 2650 patients with impacted ureteral stones. World J Urol 2017;35:1497-506. 
15. Deng T, Chen Y, Liu B, et al. Systematic review and cumulative analysis of the managements for proximal impacted ureteral stones. World J Urol 2019;37:1687-701.

16. Leonardo C, Simone G, Rocco P, et al. Laparoscopic ureterolithotomy: minimally invasive second line treatment. Int Urol Nephrol 2011;43:651-4.

17. Gao ZM, Gao S, Qu HC, et al. Minimally invasive percutaneous nephrolithotomy improves stone-free rates for impacted proximal ureteral stones: A systematic review and meta-analysis. PLoS One 2017;12:e0171230.

18. Yamaguchi K, Minei S, Yamazaki T, et al. Characterization of ureteral lesions associated with impacted stones. Int J Urol 1999;6:281-5.

19. Wollin DA, Carlos EC, Tom WR, et al. Effect of Laser Settings and Irrigation Rates on Ureteral Temperature During Holmium Laser Lithotripsy, an In Vitro Model. J Endourol 2018;32:59-63.

20. Wu C, Hua LX, Zhang JZ, et al. Comparison of renal pelvic pressure and postoperative fever incidence between standard- and mini-tract percutaneous nephrolithotomy. Kaohsiung J Med Sci 2017;33:36-43.

21. Mugiya S, Ito T, Maruyama S, et al. Endoscopic features

Cite this article as: Anan G, Kudo D, Matsuoka T, Kaiho Y, Sato $M$. The impact of preoperative percutaneous nephrostomy as a treatment strategy before flexible ureteroscopy for impacted upper ureteral stones with hydronephrosis. Transl Androl Urol 2021;10(10):3756-3765. doi: 10.21037/tau-21-547 of impacted ureteral stones. J Urol 2004;171:89-91.

22. Brito AH, Mitre AI, Srougi M. Ureteroscopic pneumatic lithotripsy of impacted ureteral calculi. Int Braz J Urol 2006;32:295-9.

23. Joshi HB, Stainthorpe A, MacDonagh RP, et al. Indwelling ureteral stents: evaluation of symptoms, quality of life and utility. J Urol 2003;169:1065-9; discussion 1069.

24. Rubenstein RA, Zhao LC, Loeb S, et al. Prestenting improves ureteroscopic stone-free rates. J Endourol 2007;21:1277-80.

25. Netsch C, Knipper S, Bach T, et al. Impact of preoperative ureteral stenting on stone-free rates of ureteroscopy for nephroureterolithiasis: a matched-paired analysis of 286 patients. Urology 2012;80:1214-9.

26. Yang Y, Tang Y, Bai Y, et al. Preoperative double-J stent placement can improve the stone-free rate for patients undergoing ureteroscopic lithotripsy: a systematic review and meta-analysis. Urolithiasis 2018;46:493-9.

27. Navetta AF, Elmekresh A, Doersch K, et al. Preoperative ureteral stenting prior to ureteroscopy for management of urolithiasis does not impact the postoperative return for unplanned care. Urol Ann 2019;11:282-6. 